\title{
ABSOLUTE STRUCTURE DETERMINATION IN ABSENCE OF HEAVY SCATTERERS
}

\author{
J. GRochowsKI \\ Regional Laboratory of Physicochemical Analysis and Structural Research \\ Jagiellonian University, Ingardena 3, 30-060 Kraków, Poland
}

Methods of absolute structure determination for molecules containing only light atoms (C, H, N, O) are discussed in the paper. This experimentally difficult problem has a direct application in the structure determination of naturally originated compounds and modern chiral drugs. Several experimental methods of absolute structure determination like electron diffraction, multiple X-ray diffraction and X-ray anomalous scattering techniques are briefly discussed and compared. An application of dedicated azimuthal scan method for the case of hexamethylenetetramine is given as an illustration of this technique.

PACS numbers: $61.10 . \mathrm{Nz}, 61.10 . \mathrm{Yh}, 33.15 . \mathrm{Bh}$

\section{Introduction}

With the recent progress of crystal structure analysis, particularly with the use of new synchrotron light sources, structures of complicated living organism molecules, like viruses, enzymes, ribosoms, are within the reach of the investigation and computational methods. It is quite interesting that in spite of living in predominantly homo-chiral biosphere, problems of structural chirality determination were on the verge of the main stream of research. In the crystallographic sense, the problem consists in determination of the absolute structure, i.e. the structure of a crystal expressed in the absolute frame of reference defining the handedness.

The first introduction of the chirality problem into natural sciences came after the famous inspection by Louis Pasteur of his wine bottle, whèn he noticed that the crystals formed around the neck of the bottle were different from those that appeared at the bottom of the bottle and not superimposed, like the left and the right hand [1]. Then, it took nearly two decades after the discovery of X-ray diffraction in 1912 followed by the formulation of the famous Friedel law in 1913 [2], until physicists became interested and experimentally able to determine an absolute structure (polarity) of zinc blende in 1930 using anomalous scattering of zinc with Au $L_{\alpha_{2}}$ (Coster, Knol and Prins [3]). Asymmetry in electron diffraction pattern for the same substance $(\mathrm{ZnS})$ was first reported by Thiessen and Moliere in 1939 [4]. The Friedel law originally formulated for X-ray diffraction has been 
applied in discussion to electron diffraction (Laue [5]). The discrepancies from the Friedel law observed in the Bragg case of electron diffraction for $\mathrm{ZnS}$ [6] were used in 1950 for absolute polarity determination confirming previous X-ray results, beginning the development of electronography as an alternative tool for absolute structure determination.

The first deduction of the absolute configuration of organic molecule (NaRb tartrate) using X-ray anomalous scattering was carried out by Bijvoet [7] in 1951, and since then, the method is under constant development reaching the investigation of anomalous scattering anisotropy for heavy atoms [8] and in the area of organic and macromolecular crystals to make a practical use of anomalous scattering from light and medium-weight atoms for solving phase problems and chiral discrimination [9-12]. A significant part of the experimental progress was achieved owing to tunability of synchrotron radiation (SR) beams.

Low-divergency SR beam enhanced also the development of multiple beam $\mathrm{X}$-ray diffraction as an alternative method of experimental phase determination allowing absolute structure determination. The use of three-beam diffraction for experimental phase determination, first suggested by Lipscomb [13] in 1949, elaborated by Post [14], was further developed by Hümmer and Billy [15, 16]. The use of synchrotron beam allowed Tischler and Batterman in 1984 [17] to measure the absolute structure factor of (622) reflection in germanium. Hümmer, Weckert and Bondza [18] designed and successfully used a dedicated SR beam with a six-wheel goniometer for absolute structure determination of $L$-asparagine monohydrate, measuring 3-beam Bragg diffraction profiles.

For chemistry and modern chirotechnology [19] processing products of stereospecific synthesis, biochemistry dealing with naturally originated compounds, pharmacology developing stereospecific drugs [20,21], determination of absolute crystal structure and absolute configuration of molecules are crucial from the point of view of synthesis efficiency, compound description and its potential application.

\section{Current status of measuring methods \\ 2.1. Multiple beam X-ray diffraction}

$N$-beam multiple diffraction occurs when $N-1$ lattice planes satisfy simultaneously Bragg's diffraction condition. In terms of the reciprocal space, $N-1$ reciprocal lattice vectors, each corresponding to one lattice plane set, and the reciprocal lattice origin marked by the primary beam are simultaneously on the Ewald sphere. The case of Bragg reflection from a unique lattice plane set could be described as 2-beam diffraction, since one reciprocal lattice vector $\boldsymbol{H}$ characteristic of one set of parallel lattice planes and the origin are simultaneously on the Ewald sphere. Let us consider the 3-beam diffraction case: two sets of lattice planes characterized by the lattice vectors $\boldsymbol{H}$ and $\boldsymbol{G}$ are simultaneously in the diffraction position. Consequently three waves are excited in the crystal: the incident wave propagated by the primary beam and two waves originated by Bragg reflections. The reflected waves interfere inside the crystals originating Bragg reflection characterized by reciprocal lattice vector $\boldsymbol{H}-\boldsymbol{G}$. Using another terminology, a necessary condition for such effect is that the difference between the Miller indices of two Bragg reflections must also correspond to a Bragg reflection. When such oriented crystal 
is rotated around $\boldsymbol{H}$ vector in the vicinity of three-beam diffraction position the intensity change appears due to interference - it depends on the phases of three structure factors: $F(-\boldsymbol{H}), F(\boldsymbol{G}), \boldsymbol{F}(\boldsymbol{H}-\boldsymbol{G})$ [22]. Measured triplets' phases may be used to determine the crystal structure, whereas an analysis of the $\psi$-scan profiles may provide the sign of triplet phases allowing identification of absolute structure. In principle the method is insensitive to anomalous scattering. Technically, it requires stable and well-collimated primary beam and precisely constructed crystal orienter allowing azimuthal rotation in $0.001^{\circ}$ steps. The crystals should have FWHM of 2-beam profile below $0.1^{\circ}$. Using a six-wheel goniostat installed on a Swiss-Norwegian beam line (European Synchrotron Radiation Facility - ESRF), the absolute structure of a compound containing small atomic fraction of nitrogen (acting as anomalous scatterer) with a formula $\mathrm{C}_{28} \mathrm{H}_{20} \mathrm{~N}_{2}$ has been determined (Weckert, Hümmer, Pattison, Weber, to be published). Hümmer, Schwegle and Weckert [23] measured triplet phases for small protein myoglobin using a tunable wavelength to diminish $N>3$ beam diffraction. It is worth pointing out that the experiment was carried out successfully on a crystal sample which contained three major mosaic blocks. Recently, Lee, Colella and Chapman [24] observed multiple Bragg diffraction in AlCuFe quasicrystal using $1.545 \AA$ synchrotron beam at National Synchrotron Light Source - NSLS (Brookhaven).

Multiple beam X-ray diffraction, due to its insensitivity to anomalous scattering effect, might be preferable for determining the absolute structure of crystals built of light atoms compounds. On the other hand, it requires high-intensity (SR or rotation-anode) primary beam and a dedicated, high-precision goniometer above the cost of instrumentation used for the crystal structure analysis. So far, there were no reports on the determination of the absolute structure of a compound with an a priori unknown crystal structure.

\subsection{Electron diffraction}

The breakdown of the Friedel law in electron diffraction pattern could be used for absolute structure determination. The Friedel law fails in electron diffraction not as a result of the absorption effect, but as a consequence of dynamic scattering between simultaneously excited diffracted beams. The effect for non-centrosymmetric crystals might be observed with various electron diffraction techniques: electron backscaterring Kikuchi diffraction patterns (BKDP), transmission electron microscopy in converged beam electron diffraction patterns (CBED), electron channelling pattern obtained on scanning electron microscope [25-28]. An application of electron diffraction methods for absolute structure determination of organic materials is limited by heat transfer. On the other hand, electron diffraction allows examination of very small single grains inside the crystalline materials not accessible for a standard X-ray diffraction. A comprehensive discussion of electron diffraction applied to organic materials is given in Ref. [29].

\subsection{X-ray anomalous scattering}

The use of anomalous scattering during diffraction experiments appears to be a method of choice in case when the investigated structure is not known a priori, 
especially that the method is not very demanding with respect to the quality of crystal [30].

Historically, two strategies have been developed. The first one assumes that handedness of molecules appearing in non-centrosymmetric crystals leaves its fingerprints in the whole diffraction pattern. During refinement of the structural model, an assumption about handedness is made and verified by statistical tests [31], the so-called global enantiomer correctness coefficient [32] or enantiomorph polarity estimation [33, 34]. In spite of its efficiency with medium-weight atoms (from $\mathrm{F}$ to $\mathrm{Ca}$ ) acting as anomalous scatterers the results of global enantiomer correctness indicators are biased with high estimated standard deviations (e.s.d.) when dealing with light atoms $(\mathrm{O}, \mathrm{N})$ as anomalous scatterers [35, 36]. In addition to that, a choice of wrong enantiomer during least-squares refinement could lead to a false and irreversible minimum [37]. In such case, a more refined approach is proper: with the knowledge of basic crystal structure (whether the absolute structure is assigned correctly or not) the set of reflections is separated into dispersion insensitive (centrosymmetric ones or with negligible dispersion component) and a subset with the largest dispersion component. As a primary criterion, measurable discrepancy from the Friedel law is used. Dispersion-sensitive reflections are carefully remeasured and grouped into the so-called Bijvoet pairs, i.e. pairs of reflections which are symmetry-related and in spite of that show an intensity difference. The handedness is deduced by comparison of inequalities involving calculated and measured intensities of Bijvoet pairs. Since usually not all inequalities signs are in agreement, appropriate discrepancy indices are calculated to verify the choice of handedness [38, 39].

For a crystal composed of molecules containing only nitrogen as the heaviest atom, the problem could be approached in two ways:

(1) With the use of wavelengths 3-7 $\AA$ synchrotron radiation in diffraction experiment, as proposed by Stuhrmann and Lehmann [40], the distance on the energy scale between $K$ absorption edge for nitrogen $(30.99 \AA)$ and the energy of the incident radiation decreases with a simultaneous increase in dispersion terms. However, it is done at the expense of a drastic decrease in the Ewald sphere radius, and consequently, spatial resolution of the diffraction pattern. It is particularly painful for small unit cells. For example, for hexamethylenetetramine only 6 reflections are accessible with a $4 \AA$ wavelength. It should be stressed, however, that the method works well for macromolecular structures, for which it was developed and may be used for known structure handedness verification in case of smaller unit cells.

(2) Employing a dedicated azimuthal scan method [12] which uses information carried out by azimuthal scan curve instead of a single number - reflection intensity in two-beam case. Azimuthal scan ( $\psi$-scan) consists of a rotation of the crystal sample around the diffraction vector and recording of the resulting diffracted intensity variation as a function of the rotation angle $\psi$. In general, instead of the Bijvoet inequality attributed to two measured intensities, two curves recorded for individual pair components are compared over selected azimuthal angle range. As a reference, azimuthal scans of centrosymmetric reflection pairs, where intensities should be equal, are also recorded.

A comprehensive discussion of this procedure including systematic errors 
treatment is given in Ref. [41]. The analysis of azimuthal scan recordings for the Bijvoet pairs of several crystal samples with weak anomalous scatterers clearly shows the reason why usually not all individual signs of the Bijvoet inequalities between intensities measured at one discrete $\psi$ value are consistent with the model predictions. The curves often intersect due to random positioning errors or the influence of the Renninger effect.

It is worthwhile to summarize the difference of experimental strategy of recording $\psi$-scan profile in X-ray multibeam method and dedicated azimuthal scan using anomalous scattering.

In a multibeam case, the investigated vicinity of $N$-beam (in practice $N=3$ ) diffraction position is narrow (about $0.1^{\circ}$ ). Consequently, $\psi$-angle increments required to a smooth construction of multibeam profile are very small (about $0.001^{\circ}$ ). Outside this narrow region, a multibeam case degenerates to a 2-beam case. Measurements are done for chosen strongly reflecting planes $\boldsymbol{H}$ and $\boldsymbol{G}$. The choice of a wavelength is implied by the resolution.

The aim of the experiment with dedicated azimuthal scan method is to prove the intensity difference of two reflections of $\boldsymbol{H}$ and $\boldsymbol{H}$ lattice planes. The investigated $\psi$-angle region is larger than in the previous case, even up to the periodicity limit $180^{\circ}$, consequently the $\psi$ increment is larger (about $0.5^{\circ}$ ). Unavoidable regions of multibeam interference rather disturb ordinate differences of two curves. Lattice planes selected for experiments have a weak reflecting power and a relatively large anomalous scattering component. The wavelength is optimized to increase anomalous scattering, however azimuthal scan methods might be successfully employed without loss of resolution using $\mathrm{Co}$ and $\mathrm{Cu}$ wavelengths.

Recently, an IUCr project absolute light-atoms-structure determination (ALASD) was undertaken in order to compare feasibility and reliability of various methods of absolute structure determination. The outcome of the project scheduled for 1994-97 will provide the state-of-the-art of measuring techniques.

\section{Sample quality examination}

The use of compatible quality samples (usually the samples of common origin) during absolute structure determination done with various measuring techniques implies the necessity of sample characterization giving rise to the question how to unambiguously quantify the crystal quality for diffraction experiment. The most natural criterion appears to be crystal mosaicity, which describes angular spread of the different small perfect crystal blocks constituting a bulk crystal sample. Smaller mosaicity is reflected in the narrower profiles of measured Bragg reflections and higher peak-to-background ratio. It results in larger diffraction limits. The use of highly collimated synchrotron radiation is ideal for testing mosaicity of crystals diminishing the masking effect caused by the primary beam divergence and spectral distribution.

Mosaicity measurements are done using the single axis oscillating crystal technique for selected reflections. The oscillating axis is perpendicular to the direct beam and the reflection is recorded in the vertical plane, where the divergence of the synchrotron beam is minimal $[42,43]$.

Another important question arises how to assess crystal quality and to mea- 
sure its mosaic spread using conventional laboratory sources. This problem is of utmost importance in preparing and selecting crystal samples for time-constrained synchrotron radiation experiments. Recently a new method has been proposed to overcome this problem with the use of low-angle acceptance primary beam monochromators. Two-channel cut monochromators in Bartels configuration provide a highly monochromated primary beam with extremely low divergence in the monochromator diffraction plane. Such a device has been designed and tested [44], proving to be efficient in spite of unavoidable time consumption.

\section{Feasibility study of X-ray anomalous scattering method}

The majority of naturally originated compounds, as well as their synthetic replicas, are built of light atoms (H, C, N, O). Since absorption edges of those light atoms are quite far from the useful range of radiation in a diffraction experiment and their relative dispersion corrections are small; absolute structure determination in such cases presents an experimental problem [45], closely related to small phase rotation occurring in macromolecular crystals $[11,46]$. In the statistical sense, a prediction of feasibility of absolute structure determination could be done starting from chemical composition of the unit cell and energy of the incident radiation. Several feasibility criteria have been developed estimating the Bijvoet ratio (i.e. the Bijvoet difference normalized by average pair intensity) prior to the experiment [47-49]. It is worth noticing that they work equally well for the case of light atoms anomalous scatterers incorporated in small molecule crystals, and for medium anomalous scatterers in macromolecular crystals. A comprehensive overview of feasibility criteria, including extension of Parthasarathy's tables of expectation values of the Bijvoet ratio for oxygen, nitrogen and carbon was given in Ref. [50].

As it may be calculated in a feasibility study, the use of nitrogen as an anomalous scatterer, i.e. chirality marker, even against the background of lighter atoms, in the most favourable stoichiometric ratio ensuring an equal scattering power of the normal and the anomalous scatterers, presents a serious experimental challenge. For example, with Copper radiation, the expected Bijvoet ratio for two ALASD standards - borazone BN, the estimated Bijvoet ratio does not exceed $0.9 \%$, whereas for hexamethylenetetramine $\mathrm{C}_{6} \mathrm{H}_{12} \mathrm{~N}_{4}$ does not exceed $0.8 \%$ [50] (both values calculated for $\sin \theta / \lambda=0.5$ ) requiring a perfect structural model and accurate intensity measurements.

\section{Absolute structure determination for hexamethylenetetramine}

The determination of the absolute structure of hexametylenetetramine (HMT) illustrates the procedure including the most sensitive Bijvoet pairs selection and technical details of measurements.

HMT $\mathrm{C}_{6} \mathrm{H}_{12} \mathrm{~N}_{4}$ crystallizes in a form of a rhombic dodecaheder $\{110\}$, in a cubic crystal system, space group $I-43 m, a=7.028(1) \AA$. The structure (Fig. 1) was first determined by Becka and Cruickshank [51] from photographic data and later by many authors [ 52 and references therein]. Basing on $\mathrm{Cu} K_{\alpha}$ single crystal diffractometric data of $0.81 \AA$ resolution several structural models were refined. Following various refined models, sets of the most sensitive Bijvoet pairs were 


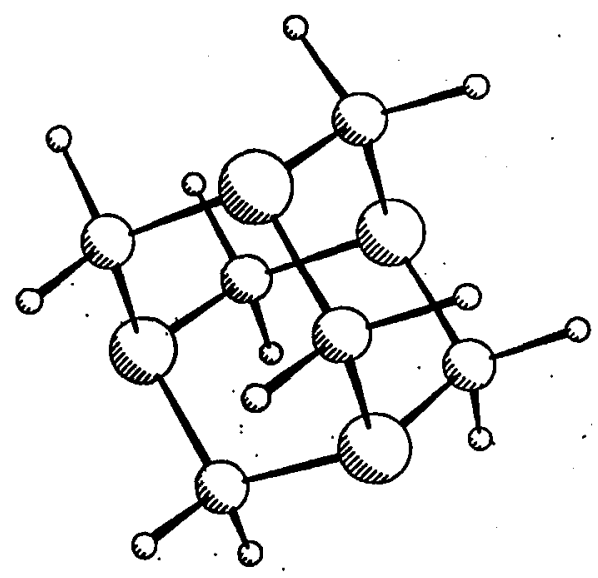

Fig. 1. A perspective drawing of a HMT molecule.

selected. For conventional and multipole [53] models the Bijvoet inequalities were congruent (Table I).

For an azimuthal scan analysis two pairs of weak reflections with significant dispersion component $(411,-4-1-1)$ and $(3-45,-34-5)$ were chosen with a third centrosymmetric pair $(604,-60-4)$ taken as a reference. The choice of a particular sign configuration of Miller indices relevant to a sample orientation was

TABLE I

Most dispersion-sensitive Bijvoet pairs for HMT, copper radiation. The values of the Bijvoet ratio are model-dependent. Observability is defined as the Bijvoet difference divided by the average e.s.d of the given Friedel pair.

\begin{tabular}{|c|c|c|c|c|c|c|c|c|c|}
\hline \multirow{2}{*}{\multicolumn{3}{|c|}{$\begin{array}{c}\text { Miller } \\
\text { indices }\end{array}$}} & \multicolumn{3}{|c|}{ Multipole model } & \multicolumn{3}{|c|}{ Conventional model } & \multirow{2}{*}{$\begin{array}{c}\text { Observ- } \\
\text { ability } \\
(\times 100)\end{array}$} \\
\hline & & & $I(+)$ & $I(-)$ & Bijvoet & $I(+)$ & $I(-)$ & Bijvoet & \\
\hline 1 & 1 & 2 & 420.783 & 423.207 & -5.7 & 432.432 & 434.431 & -4.6 & 52 \\
\hline 1 & 1 & 4 & 0.994 & 1.047 & -51.9 & 1.014 & 1.061 & -45.3 & 78 \\
\hline 1 & 1 & 6 & 21.678 & 21.548 & 6.0 & 22.203 & 22.099 & 4.7 & 24 \\
\hline 1 & 2 & 7 & 15.171 & 15.047 & 8.2 & 15.272 & 15.148 & 8.2 & 39 \\
\hline 1 & 3 & 6 & 17.115 & 16.958 & 9.2 & 17.766 & 17.598 & 9.5 & 45 \\
\hline 2 & 2 & 6 & 92.064 & 91.336 & 7.9 & 93.799 & 93.123 & 7.2 & 49 \\
\hline 2 & 3 & 7 & 14.205 & 14.108 & 6.9 & 15.054 & 14.961 & 6.2 & 30 \\
\hline 3 & 3 & 4 & 3.960 & 4.036 & -19.0 & 3.834 & 3.869 & -9.1 & 20 \\
\hline 3 & 3 & 6 & 21.949 & 21.800 & 6.8 & 21.086 & 20.949 & 6.5 & 26 \\
\hline 4 & 5 & 0 & 0.588 & 0.567 & 36.4 & 0.757 & 0.733 & 32.2 & 34 \\
\hline
\end{tabular}


imposed by the uniform sample illumination condition by the primary beam and accessibility of symmetrical azimuthal angle range for kappa-geometry CAD-4 diffractometer. Measurements were carried out using $\mathrm{Cu} K_{\alpha_{1}}(\lambda=1.54052 \AA)$ and Co $K_{\alpha_{1}}(\lambda=1.78896 \AA)$ radiation, allowing us to use the same monochromators setup (graphite (002), Ge (111)). Anomalous dispersion corrections for nitrogen with both radiation types have the values $\delta f^{\prime \prime}=0.0180$ and $\delta f^{\prime \prime}=0.0248$, respectively. Prior to azimuthal scan recording, careful 2D reflection mapping was carried out using graphite (002) flat monochromator, $\mathrm{Ge}(111)$ flat monochromator, $\mathrm{Ge}(220)$ channel cut monochromator and $\mathrm{Ge}(220)$ Bartels type monochromator. The reflection mapping allowed for detection of non-uniform mosaicity and, for good samples, a rational choice of scan direction and scan angular range (Fig. 2). The choice of $\psi$ angle range and increment $\left(-40\right.$ to $40^{\circ}$ and increment $\left.0.5^{\circ}\right)$ was a necessary compromise imposed by HMT instability at room temperature due to sublimation and possible radiation damage. The recorded azimuthal scan curves (Figs. 3a,b,c) for dispersion sensitive pairs had the Bijvoet difference proportional to relative dispersion corrections, whereas for a pair of centrosymmetric reflections (604) and (-60-4) experimental values of the Bijvoet ratio were zero (within 3 e.s.d.) and wavelength insensitive. Table II presents a comparison of calculated and observed normalized Bijvoet differences.

The analysis of azimuthal scan curves leads to internally consistent results, as compared to the calculated enantiomorph polarity (Flack) coefficient [34], which converged to 1.59 with e.s.d. equal to 1.6 . In the ideal case, the expected values for the Flack coefficient are 0 for the correct and +1 for the inverted model.

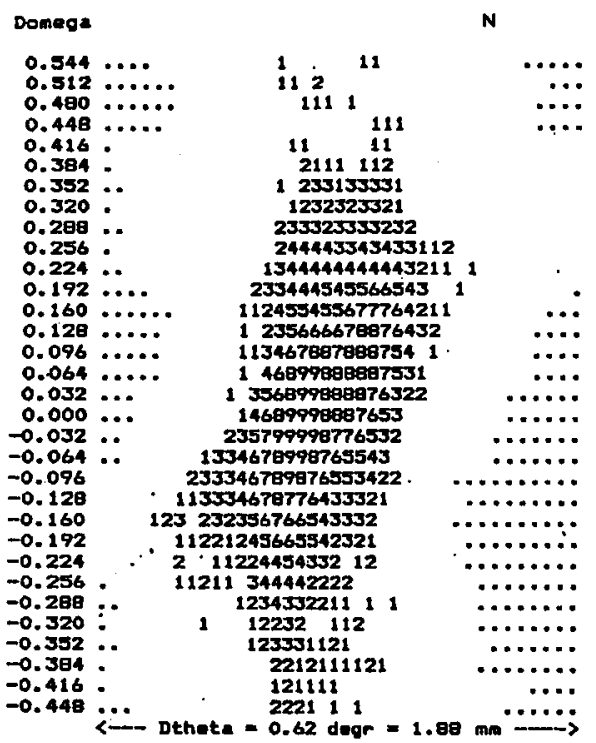

Fig. 2. Two-dimensional mapping (omega-theta plot) of a typical medium-theta reflection of HMT with visible non-uniform mosaicity. 

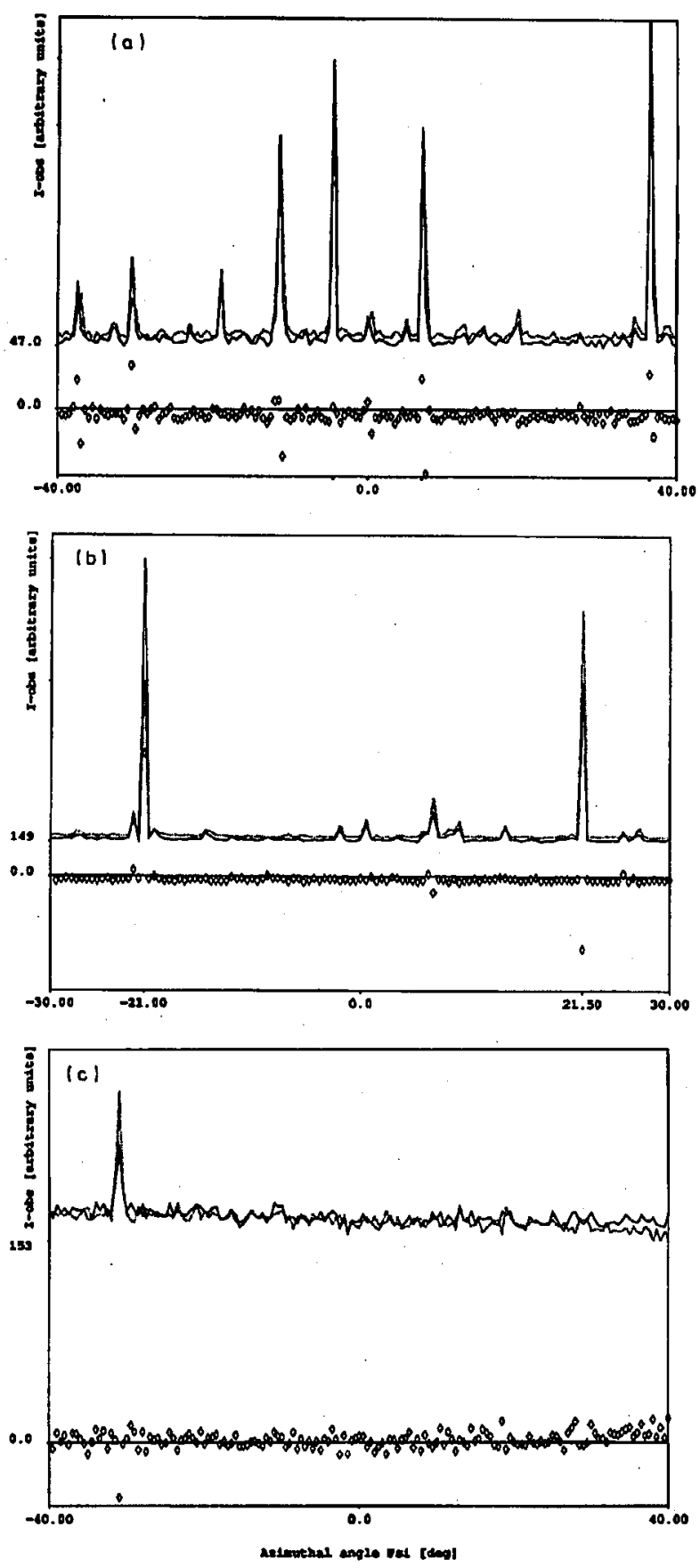

Fig. 3. Azimuthal scan curves for (a) (-4-11) Bragg reflection and its Friedel mate recorded with copper radiation, (b) (411) Bragg reflection and its Friedel mate recorded with cobalt radiation, (c) a pair of centrosymmetric reflections (604) and (-60-4) recorded with cobalt adiation. 
TABLE II

A comparison of calculated Bijvoet ratio values for HMT with those estimated from azimuthal scan profiles by statistical treatment and Fourier filtering. The values of calculated Bijvoet ratio values are model dependent and accurate numerical agreement with experimental values is not always possible.

\begin{tabular}{|c|c|c|c|c|c|c|}
\hline \multirow{4}{*}{ Friedel pair } & \multirow{4}{*}{$\begin{array}{l}\text { Radiation } \\
\text { /Wave- } \\
\text { length }[\AA]\end{array}$} & \multicolumn{3}{|c|}{ Calculated Bijvoet ratio $\Delta I / I$} & \multicolumn{2}{|c|}{ Observed Bijvoet ratio } \\
\hline & & \multirow{3}{*}{$\begin{array}{c}\text { Monopole } \\
\text { model }\end{array}$} & \multirow{2}{*}{\multicolumn{2}{|c|}{$\frac{\text { Multipoles }}{\text { program }}$}} & \multirow{3}{*}{$\begin{array}{l}\text { (statistical } \\
\text { treatment) }\end{array}$} & \multirow{3}{*}{$\begin{array}{l}\text { (Fourier } \\
\text { filtering) }\end{array}$} \\
\hline & & & & & & \\
\hline & & & VALRAY* & $\mathrm{XD}^{* *}$ & & \\
\hline$(-4-11) /(41-1)$ & $\mathrm{Cu} / 1.54178$ & -0.045 & -0.051 & -0.050 & $-0.074(19)$ & $-0.066(19)$ \\
\hline$(411) /(-4-1-1)$ & $\mathrm{Co} / 1.7902$ & -0.081 & - & -0.069 & $-0.082(14)$ & $-0.064(14)$ \\
\hline$(604) /(-60-4)$ & $\mathrm{Co} / 1.7902$ & 0.0 & 0.0 & 0.0 & $-0.005(4)$ & $0.012(4)$ \\
\hline
\end{tabular}

*R.F . Stewart, Acta Crystallogr. A 32, 564 (1976).

**T. Koritszansky, S. Howard, P.R. Mallinson, Z. Su, T. Richter, N.K. Hansen, Freie Universität, Berlin 1994.

Consequently, the absolute structure of HMT was determined with the azimuthal scan procedure with a higher confidence level.

It should be noted that the Bartels monochromator constructed for the present research provides a primary beam of low divergence comparable with synchrotron beams. Thus it allows for sample pre-examination prior to real synchrotron experiment, crucial to the investigation of naturally originated organic compounds.

\section{Acknowledgments}

The work was supported by the Committee for Scientific Research grant No. PB 1279/P4/93/04. The author is grateful to Prof. J. Auleytner for helpful discussions of a primary beam monochromator construction and to Prof. A. Kirfel for reference calculations.

\section{References}

[1] L. Pasteur, Comptes Rendus, Acad. Sci. (Paris) [séance du 15 mai, 1848] 26, 535 (1848).

[2] G. Friedel, Comptes Rendus, Acad. Sci. (Paris) 157, 1533 (1913).

[3] D. Coster, K.S. Knol, J.A. Prins, Z. Phys. 63, 345 (1930).

[4] P.A. Thiessen, K. Moliere, Ann. Phys. 34, 449 (1939).

[5] M. von Laue, Materiewellen und Thre Interferenzen, Akademische Verlagsgesellschaft Geest \& K.G. Portig, Leipzig 1948, p. 257.

[6] S. Miyake, R. Uyeda, Acta Crystallogr. 3, 314 (1950).

[7] J.M. Bijvoet, A.F. Peerdeman, A.J. van Bommel, Nature 168, 271 (1951).

[8] A. Kirfel, in: Resonant Anomalous X-Ray Scattering, Theory and Application, Eds. G. Materlik, C.J. Sparks, K. Fischer, North Holland, Amsterdam 1994, p. 231.

[9] J. Karle, in: Resonant Anomalous X-Ray Scattering, Theory and Application, Eds. G. Materlik, C.J. Sparks, K. Fischer, North Holland, Amsterdam 1994, p. 145.

[10] H.B. Sturmann, M.S. Lehmann, in: Resonant Anomalous X-Ray Scattering, Theory and Application, Eds. G. Materlik, C.J. Sparks, K. Fischer, North Holland, Amsterdam 1994, p. 175. 
[11] W.A. Hendrickson, Science 254, 51 (1991).

[12] J. Grochowski, P. Serda, Acta Phys. Pol. A 82, 147 (1992).

[13] W.N. Lipscomb, Acta Crystallogr. 2, 193 (1949).

[14] B. Post, Phys. Rev. Lett. 39, 760 (1977).

[15] K. Hümmer, H. Billy, Acta Crystallogr. A 38, 841 (1982).

[16] K. Hümmer, H. Billy, Acta Crystallogr. A 42, 127 (1986).

[17] J.Z. Tischler, B.W. Batterman, Phys. Rev. B 30, 7060 (1984).

[18] K. Hümmer, A. Weckert, H. Bondza, Acta Crystallogr. A 45, 182 (1989).

[19] R.A. Sheldon, Chirotechnology, Marcel Dekker, New York 1993, ch. 2 and 3.

[20] D.E. Drayer, Clinical Pharmacol. Therapeutics 40, 125 (1986).

[21] E.J. Ariens, Eur. J. Clin. Pharmacol. 26, 663 (1984).

[22] K. Hümmer, W. Schwegle, E. Weckert, Acta Phys. Pol. A 82, 83 (1992) and references therein.

[23] K. Hümmer, W. Schwegle, E. Weckert, Acta Crystallogr. A 47, 60 (1991).

[24] H. Lee, R. Colella, L.D. Chapman, Acta Crystallogr. A 49, 600 (1993).

[25] P. Goodman, G. Lehmpfuhl, Acta Crystallogr. A 24, 339 (1968).

[26] K.Z. Baba-Kishi, J. Appl. Cryst. 24, 38 (1991).

[27] J. Tafto, J.C.H. Spence, J. Appl. Cryst. 15, 60 (1982).

[28] P. Goodman, Acta Crystallogr. A 31, 804 (1975).

[29] Proceedings of the Symposium on Electron Crystallography, Ed. D.L. Dorset, Vol. 28, ACA Transactions, Pittsburgh 1992.

[30] H. Holloway, J. Appl. Phys. 40, 2187 (1969).

[31] W.C. Hamilton, Acta Crystallogr. 18, 502 (1965).

[32] D. Rogers, Acta Crystallogr. A 37, 734 (1981).

[33] H.D. Flack, Acta Crystallogr. A 39, 876 (1983).

[34] G. Bernardinelli, H.D. Flack, Acta Crystallogr. A 41, 500 (1985).

[35] J. Grochowski, M. Rutkowska, B. Rys, P. Serda, G. Snatzke, Chem. Ber. 125, 1837 (1992).

[36] J. Grochowski, P. Serda, V. Kupcik, K. Eichhorn, in: HASYLAB Jahresbericht 1989, Ed. G. Materlik, DESY - HASYLAB, Hamburg 1989, p. 381.

[37] P.G. Jones, in: Crystallographic Computing 3, Eds. G.M. Sheldrick, C. Krüger, R. Goddard, Clarendon Press, Oxford 1985, p. 260.

[38] M. Martinez-Ripoll, J. Fayos, Z. Krist. 152, 189 (1980).

[39] G. Beurskens, G.J.H. Noordik, P.T. Beurskens, Crystallogr. Struct. Commun. 9, 23 (1980).

[40] H.B. Stuhrmann, M.S. Lehmann, in: Resonant Anomalous X-Ray Scattering, Eds. G. Materlik, C.J. Sparks, K. Fischer, North-Holland, Amsterdam 1994, p. 175.

[41] J. Grochowski, P. Serda, Acta Phys. Pol. A 91, No. 5 (1997), in press.

[42] S. Weisgerber, J.R. Helliwell, E. Weckert, K. Hoelzer, K. Schroer, Acta Crystallogr. D 51, 1099 (1995).

[43] J.L. Ferrer, J. Hirschler, M. Roth, J.C. Fontecilla-Camps, ESRF Newsletter 26, 27 (1996). 
[44] J. Grochowski, P. Serda, W. Paszkowicz, to be published.

[45] J. Grochowski, P. Serda, in: Inter. Conf. on Anomalous Scattering, Malente (Hamburg), August 17-21, 1992, Book of Abstracts, P6.

[46] S.R. Hubbard, R.J. Greenall, M.M. Woolfson, Acta Crystallogr. D 51, 979 (1995).

[47] W.H. Zachariasen, Acta Crystallogr. 18, 714 (1965).

[48] W.A. Hendrickson, Science 254, 51 (1991).

[49] S. Parthasarathy, Acta Crystallogr. 22, 98 (1967).

[50] J. Grochowski, P. Serda, Chirality 5, 277 (1993).

[51] L.N. Becka, D.W.J. Cruickshank, Proc. R. Soc. (London) A 273, 435 (1963).

[52] J. Grochowski, P. Serda, K. Wilson, Z. Dauter, J. Appl. Crystallogr. 27, 722 (1994).

[53] A. Kirfel, private communication. 